\title{
Segmental Arteries and Veins at Higher Lumbar Levels Can Intersect the Adjacent Caudal Intervertebral Disc in the Anterior Part of the Spinal Column: A Cadaveric Analysis
}

\author{
Kiyoshi Yagi, Nobuyuki Suzuki, Jun Mizutani, Kenji Kato, \\ Akira Kondo, Yuya Waseda, Yuta Goto, Hideki Murakami \\ Department of Orthopaedic Surgery, Graduate School of Medical Sciences, Nagoya City University, Nagoya, Japan
}

\begin{abstract}
Study Design: A cadaveric study.
Purpose: To investigate the anatomical features of segmental arteries and veins in the anterior part of the spinal column to prevent segmental vessel injury.

Overview of Literature: The lateral transpsoas approach to the lumbar intervertebral discs (IVD) is associated with the risk of segmental vessel injury. Previous studies have described the vascular anatomy on the lateral part of the vertebral body. However, there are no studies that describe the segmental vessels on its anterior aspect. Here, we report the important anatomical features of the segmental arteries and veins that can intersect the anterior part of the IVD. These vessels are considered at risk of vascular injury when placing the anterior retractors during lateral lumbar interbody fusion or cutting the anterior longitudinal ligament during anterior column realignment.

Methods: Five formalin-embalmed human cadavers were used. We assessed the proportion of segmental arteries and veins that intersected the IVD in the $L 2-\mathrm{L} 5$ range and their course on the anterior part of the spinal column.

Results: The segmental arteries and veins commonly intersect the anterior part of the IVD (artery, 28.1\%; vein, 42.1\%). Seven of 10 $(70 \%)$ segmental arteries at L2 intersected the IVD, but only one artery intersected the IVD at L3 and L4. The proportions of segmental veins that intersected the IVD were $60 \%, 50 \%$, and $16.7 \%$ at $L 2, L 3$, and $L 4$, respectively.

Conclusions: The segmental arteries and veins frequently intersect the IVD in the anterior part of the spinal column. Therefore, it is necessary to consider these individual anatomical features to prevent vascular damage during lateral lumbar interbody fusion surgery.
\end{abstract}

Keywords: Segmental artery; Segmental vein; Lateral lumbar interbody fusion; Anterior column realignment; Intervertebral disc

\section{Introduction}

Minimally invasive techniques for spinal surgery (MIS) have been developed to reduce tissue damage. MIS enable surgeons to approach the vertebral bodies and intervertebral discs (IVD) with minimal tissue injury and exposure,

Received Aug 20, 2020; Revised Dec 8, 2020; Accepted Dec 8, 2020

Corresponding author: Nobuyuki Suzuki

Department of Orthopaedic Surgery, Graduate School of Medical Sciences, Nagoya City University, 1 Kawasumi, Mizuhocho, Mizuhoku, Nagoya, 467-8601, Japan

Tel: +81-52-853-8236, Fax: +81-52-842-0266, E-mail: nobuyuki.suzuki@me.com 
using specially designed devices. The technique used for lateral lumbar interbody fusion (LLIF) allows exposure of the anterior elements of the spine and has become a major option in the treatment of degenerative disk disease, spinal stenosis, degenerative scoliosis, and spondylolisthesis [1-4]. LLIF is a useful method in achieving effective fusion and indirect decompression of the intervertebral foramen and spinal canal [3-9]. Moreover, anterior column realignment (ACR) through the lateral transpsoas approach, with release of the anterior longitudinal ligament and annulus, is also widely performed to correct focal kyphotic deformity [10-12]. However, these new techniques are associated with risks of severe complications, such as bowel, ureteric, vascular, or lumbar nerve injuries [6,13-15]. Vascular injury can be fatal because it is difficult to manage in the limited operative window provided by LLIF. Fujibayashi et al. [13] reported that segmental artery injuries occurred in $0.4 \%$ of 2,997 LLIF cases. Prevention of these complications requires a more precise understanding of the regional and focal anatomy of the spine than that needed when using conventional surgical techniques.

To date, several studies have analyzed the vascular anatomy of the lateral part of the vertebral body to prevent segmental artery injury [16-19]. Nojiri et al. [18] reported the anatomical features of the segmental arteries and their branches that intersect the IVD on the lateral part of the vertebral body. Heo et al. [19] reported that threedimensional (3D) reconstructions from spinal computed tomography (CT) angiography were useful in evaluating the anatomy of the segmental arteries. However, little has been reported about segmental veins. Furthermore, the detailed vascular anatomy of the anterior part of the spinal column is yet to be described.

This study aimed to investigate the segmental arteries and veins in the anterior aspect of the spinal column. We report important anatomical features of the segmental arteries and veins of the higher-level lumbar vertebrae that can intersect the anterior part of the IVDs. We consider that these vessels are at risk of vascular injury when placing anterior retractors during LLIF or incising the anterior longitudinal ligament during ACR.

\section{Materials and Methods}

\section{Subjects and materials}

Five formalin-embalmed human cadavers, one man and four women, with an average age of 85.2 years (range, 67-
105 years), were used in this study. All specimens showed degenerative changes of the spine, including the presence of vertebral osteophytes, ossification of the annulus fibrosus, and degenerative scoliosis, but did not have a history of abdominal or spinal surgery.

\section{Dissection}

Each cadaver was placed in the supine position, and a midline abdominal incision was made. The abdominal viscera were resected, and the retroperitoneal area was exposed. The abdominal aorta and inferior vena cava were identified; then, the psoas muscles and lumbar plexus were dissected to expose the lumbar vertebrae (L2-S1). The segmental arteries and veins were identified by tracing them from their connection to the abdominal aorta and inferior vena cava, respectively. The segmental arteries and veins from L2 to L5 were evaluated. The anterior part of the vertebral body was defined as the $180^{\circ}$ ventral side of the vertebral body.

\section{Evaluation}

First, the proportion of segmental vessels out of the identified vessels that intersected the IVD was evaluated. Second, the branch angle on the caudal side between the segmental vessels and abdominal aorta or inferior vena cava was measured with a protractor (Fig. 1). Third, of the segmental vessels that intersected the IVD, the cranial/caudal distance between the origin of the segmental vessels from abdominal aorta or inferior vena cava and the midpoint of the caudal IVD with a ruler (Fig. 1). Finally, the horizontal distance between the lateral edge of the caudal IVD and intersection point of the vessels was measured with a ruler (Fig. 1). All analyses were performed using open-source statistical computing software (R package; http://www.r-project.org/). One-way analysis of variance (ANOVA) and post hoc Tukey-Kramer test were used to compare the branch angle between the segmental vessels and abdominal aorta or inferior vena cava at each level. One-way ANOVA was also used to analyze the distance between the origin of the segmental vessels and midpoint of the caudal IVD and the distance between the lateral edge of the caudal IVD and intersection point of the vessels at each level. Fisher's exact test was used to compare the proportion of the right and left segmental vessels that intersected the IVD and the relationship between the ori- 


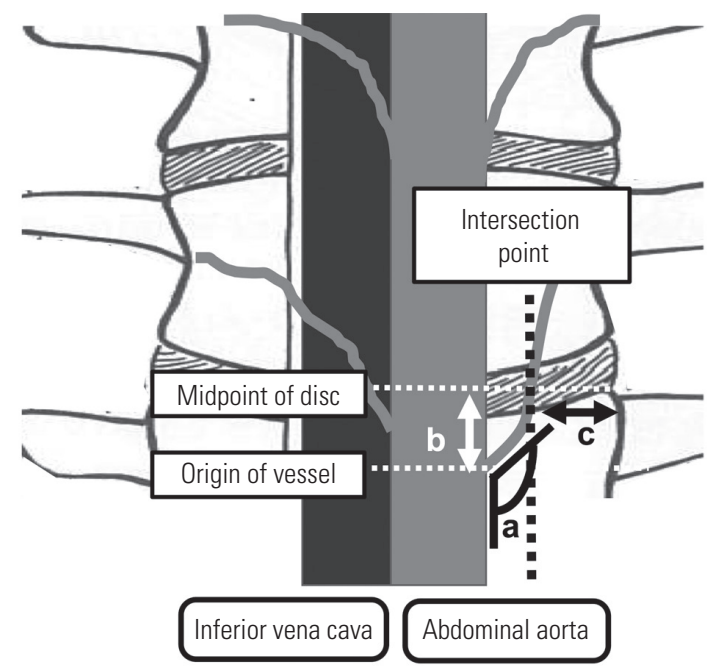

Fig. 1. Measured parameters. a: branching angle of the segmental vessels; b: cranial/caudal distance from the origin of the segmental vessels to the intervertebral disc (IVD); c: horizontal distance from the lateral edge of the IVD's to the segmental vessels on the IVD.

gin of the segmental vessels and vertebral body. Student $t$-test was used to compare the right and left horizontal distances between the segmental vessel intersection point and lateral edge of the IVDs. Statistical significance was set at a $p$-value $<0.05$.

\section{Ethical approval}

The present study was approved by the institutional review board of Nagoya City University on $16 \mathrm{Au}-$ gust 2018 (approval no., 60-18-0066).

\section{Results}

\section{Morphological relationships between the segmental vessels and caudal adjacent IVDs}

We used five specimens to investigate the proportion at which the segmental vessels intersected the IVD. We identified all bilateral segmental arteries at L2, L3, and L4 but only identified two segmental arteries at L5. Usually, the L5 segmental artery was not identified because of the difference in arterial supply, as the L5 lamina is supplied by either the L4 segmental artery or the iliolumbar artery [20]. In this study, we found the aortic bifurcation below the L5/S1 disk level in one cadaver; thus, we could identify two L5 segmental arteries. A total of 32 segmental arteries (L2, 10; L3, 10; L4, 10; L5, 2) were identified. Seven
Table 1. Number of identified segmental vessels and the rate at which they intersect the IVD

\begin{tabular}{|c|c|c|c|c|}
\hline \multirow{2}{*}{ Level } & \multicolumn{2}{|c|}{ Artery } & \multicolumn{2}{|c|}{ Vein } \\
\hline & Identified & Intersected IVD & Identified & Intersected IVD \\
\hline L2 & $10(100)$ & $7(70)$ & $5(50)$ & $3(60)$ \\
\hline L3 & $10(100)$ & $1(10)$ & $8(80)$ & $4(50)$ \\
\hline L4 & $10(100)$ & $1(10)$ & $6(60)$ & $1(16.7)$ \\
\hline L5 & $2(20)$ & 0 & 0 & 0 \\
\hline Total & 32 & $9(28.1)$ & 19 & $8(42.1)$ \\
\hline
\end{tabular}

Values are presented as number (\%). IVD, intervertebral discs.

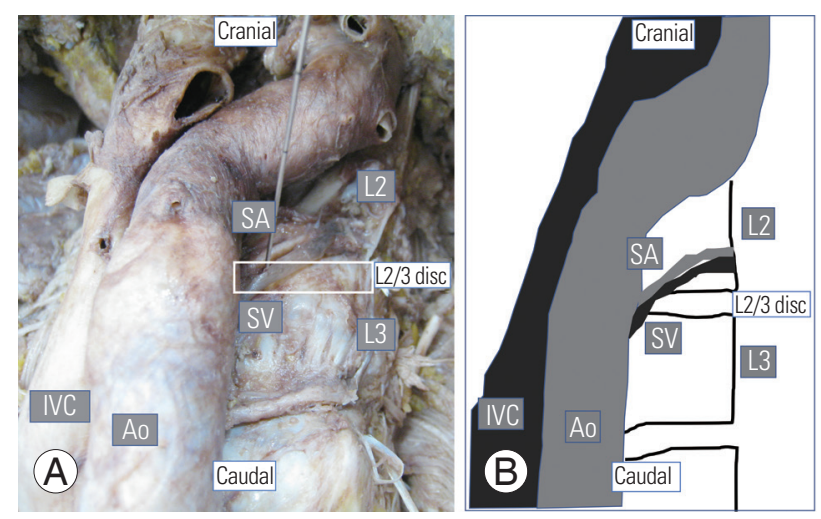

Fig. 2. Photograph (A) and illustration (B) showing the L2 segmental artery and vein intersecting the intervertebral disc (IVD) in the anterior part of the spinal column. SA, segmental artery; SV, segmental vein; A0, abdominal aorta; IVC, inferior vena cava.

of $10(70 \%)$ segmental arteries at L2 intersected the IVD, whereas only one artery intersected the IVD at L3 and L4 (10\% each). However, only a total of 19 segmental veins (L2, 5; L3, 8; L4, 6; L5, 0) could be identified. The proportions of the segmental veins that intersected the IVD were $60 \%, 50 \%$, and $16.7 \%$ at $\mathrm{L} 2, \mathrm{~L} 3$, and $\mathrm{L} 4$, respectively. The overall proportions of the segmental arteries and veins that intersected the IVD were $28.1 \%$ and $42.1 \%$, respectively (Table 1). Fig. 2 provides a cadaveric photograph and illustration of the L2 segmental artery and vein that intersect the IVD in the anterior part of the spinal column.

In comparing the left and right sides, of 16 cases, six right segmental arteries (37.5\%) and three left segmental arteries $(18.8 \%)$ intersected the IVD $(p=0.23)$. Of nine cases, five right segmental veins $(55.6 \%)$ and three left segmental veins $(30.0 \%)$ intersected the IVD $(p=0.25)$. Therefore, there was no statistically significant difference 
between the left and right sides, although the segmental vessels tended to intersect the IVD more often on the right side.

\section{Segmental vessel course in the anterior part of the spinal column}

We measured several parameters to investigate the course of the segmental vessels. We investigated how segmental vessels branched from the abdominal aorta and measured the caudal angle between the segmental vessels and aorta or inferior vena cava. The branch angle of the segmental arteries and veins at each vertebral level gradually decreased from L2 to L5. The higher-level lumbar segmental

Table 2. Branch angle between the segmental vessels and the abdominal aorta or the inferior vena cava

\begin{tabular}{lcc} 
Level & Artery $\left({ }^{\circ}\right)$ & Vein $\left({ }^{\circ}\right)$ \\
\hline L2 & $119 \pm 25.4$ & $130 \pm 21.0$ \\
L3 & $111 \pm 16.4$ & $121 \pm 10.0$ \\
L4 & $97 \pm 17.9$ & $118 \pm 17.7$ \\
L5 & $75 \pm 5.0$ & $\mathrm{NA}$ \\
\hline
\end{tabular}

Values are presented as mean \pm standard deviation.

NA, not applicable. arteries steeply branched toward the cranial side. Two L5 segmental arteries branched toward the caudal side from the aorta, and the branch angles of these arteries were $<90^{\circ}$. This tendency was shown to be statistically significant by one-way ANOVA ( $p=0.03$ ), but there was no statistically significant difference using a post hoc TukeyKramer test. This pattern was similar in veins (Table 2), although there was no statistically significant difference in the result of the one-way ANOVA. We also measured the cranial/caudal distance between the origin of the segmental vessels and caudal adjacent IVD. At higher lumbar levels, more segmental vessels appeared to branch at the vertebra situated one level below ( $7 / 10$ at the L2 artery, 3/5 at the $\mathrm{L} 2$ vein, and $4 / 8$ at the $\mathrm{L} 3$ vein) (Table 3). In cases in which the branch originates at the vertebra one level below, the cranial/caudal distance between the origin of the segmental vessels and caudal adjacent IVD was $5.7 \pm 4.2$ $\mathrm{mm}$ at the L2 arteries $(\mathrm{n}=7), 15.9 \pm 2.5 \mathrm{~mm}$ at the $\mathrm{L} 2$ veins $(\mathrm{n}=3)$, and $2.3 \pm 0.6 \mathrm{~mm}$ at the $\mathrm{L} 3$ veins $(\mathrm{n}=4)$, respectively (Table 4), and these were not statistically significant.

\section{Intersection point between segmental vessels and caudal adjacent intervertebral discs}

We also investigated the horizontal distance of the inter-

Table 3. The relationship between the origin of the segmental vessels and each vertebral body

\begin{tabular}{|c|c|c|c|c|c|c|}
\hline \multirow{2}{*}{ Branch origin } & \multicolumn{3}{|c|}{ Artery } & \multicolumn{3}{|c|}{ Vein } \\
\hline & One level below vertebra & Same vertebra & $p$-value & One level below vertebra & Same vertebra & $p$-value \\
\hline L2 & $7(70.0)$ & $3(30.0)$ & 0.0018 & $3(60.0)$ & $2(40.0)$ & 0.68 \\
\hline L3 & $1(10.0)$ & $9(90.0)$ & 0.27 & $4(50.0)$ & $4(50.0)$ & 1.0 \\
\hline$\llcorner 4$ & $1(10.0)$ & $9(90.0)$ & 0.27 & $1(16.7)$ & $5(83.3)$ & 0.30 \\
\hline L5 & 0 & $2(100.0)$ & 0.92 & NA & NA & NA \\
\hline
\end{tabular}

Values are presented as number (\%).

NA, not applicable.

Table 4. Distance between the origin of the segmental vessels and midpoint of the caudal intervertebral disc at each vertebral level

\begin{tabular}{|c|c|c|c|c|}
\hline \multirow{2}{*}{ Branch origin } & \multicolumn{2}{|c|}{ Artery (mm) } & \multicolumn{2}{|c|}{ Vein (mm) } \\
\hline & One level below vertebra & Same vertebra & One level below vertebra & Same vertebra \\
\hline L2 & $5.7 \pm 4.2$ & $10.8 \pm 3.1$ & $15.9 \pm 2.5$ & $8.9 \pm 7.7$ \\
\hline L3 & $2.0 \pm 0$ & $8.9 \pm 6.2$ & $2.3 \pm 0.6$ & $4.6 \pm 2.0$ \\
\hline L4 & $2.0 \pm 0$ & $12.3 \pm 4.0$ & $3.0 \pm 0$ & $4.1 \pm 2.3$ \\
\hline L5 & NA & $11.0 \pm 6.0$ & NA & NA \\
\hline
\end{tabular}

Values are presented as mean \pm standard deviation.

NA, not applicable. 


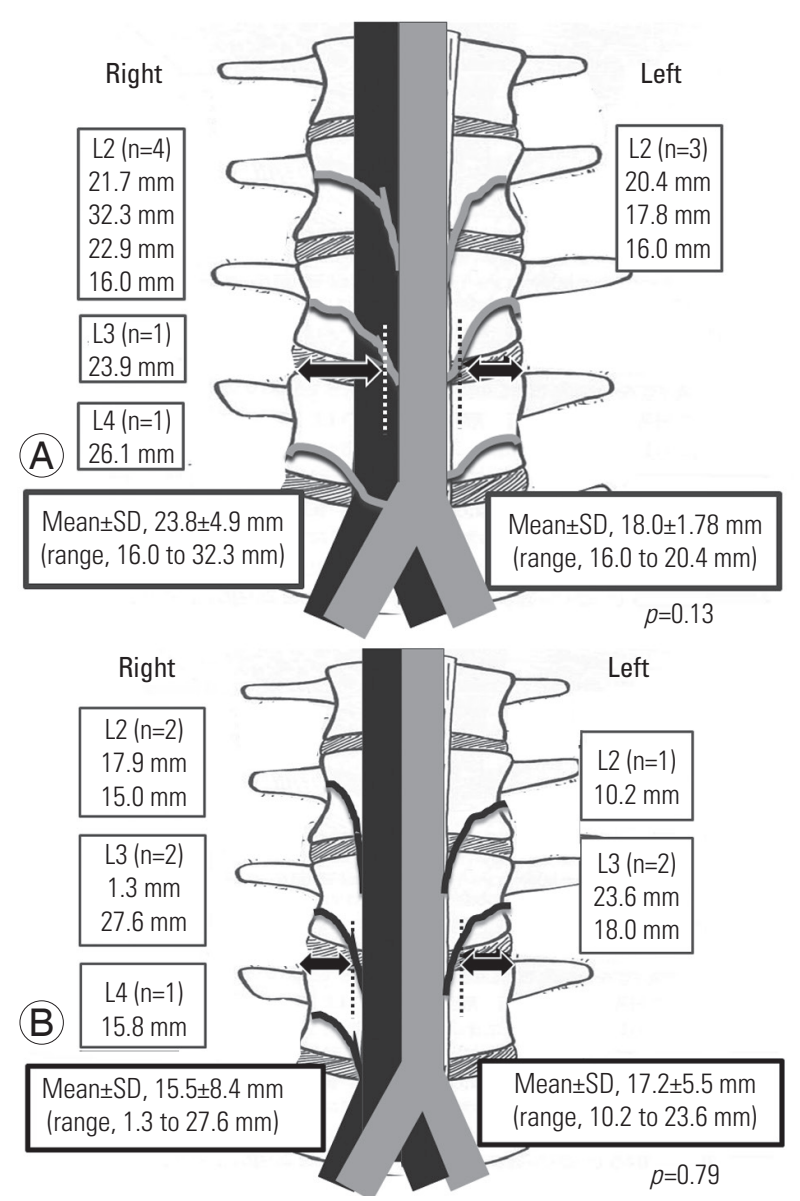

Fig. 3. Measured values of each horizontal distance from the side of the intervertebral disc (IVD) to the intersection point of the segmental vessels at each vertebral level, in those cases in which the segmental vessels intersect the IVD. (A) Segmental artery. (B) Segmental vein. SD, standard deviation.

section points between the segmental vessels and lateral edge of the IVD, as this distance indicates how close segmental vessels are to the surgical site when enlarging the surgical window using a retractor. Fig. 3 shows the measured values of each horizontal distance from the lateral edge of the IVD to the intersection point of the segmental vessels at each vertebral level, in cases where this intersection occurred. For segmental arteries, the mean horizontal distance was $23.8 \pm 4.9 \mathrm{~mm}$ (range, $16.0-32.3 \mathrm{~mm}$ ) on the right side and $18.0 \pm 1.78 \mathrm{~mm}$ (range, $16.0-20.4 \mathrm{~mm}$ ) on the left side $(p=0.13)$ (Fig. 3A). For segmental veins, the mean horizontal distance was $15.5 \pm 8.4 \mathrm{~mm}$ (range, $1.3-27.6 \mathrm{~mm}$ ) on the right side and $17.2 \pm 5.5 \mathrm{~mm}$ (range, $10.2-23.6 \mathrm{~mm}$ ) on the left side ( $p=0.79$ ) (Fig. 3B). There was no statistically significant difference between the right and left sides in relation to this horizontal distance.

\section{Discussion}

LLIF and ACR are useful surgical methods in providing mechanical stability, preservation of back muscles, and indirect decompression of the spinal canal [1-5]; however, there are some critical risks, such as injury to the segmental vessels that could result in a hematoma or other hemorrhagic complications. Fujibayashi et al. [13] reported segmental artery injury in $0.4 \%$ of 2,997 LLIF cases. Abe et al. [21] found segmental artery injury in 2.6\% of 155 cases of oblique lateral interbody fusion. Beckman et al. [22] reported seven cases $(0.18 \%)$ of symptomatic contralateral psoas hematoma in a total of 3,950 cases. Peiro-Garcia et al. [23] revealed a case of LLIF that led to hemorrhagic shock due to a giant hematoma in the contralateral retroperitoneum.

It is necessary to fully understand the anatomy of the segmental arteries and veins in the anterior part of the vertebral column to minimize the risk of segmental vessel injury during LLIF or ACR. The course of the segmental vessels is usually simple. Two segmental arteries arise from the dorsal surface of the aorta, encircle the vertebral body, and merge on the posterior surface. At the transverse process, each artery bifurcates into an intercostal segment, which courses to the ribs, and a dorsal segment, which branches into several smaller vessels that proceed to the posterior elements and pass through the neural foramen into the vertebral canal [24]. Alkadhim et al. [25] reported that the average distance from the segmental artery to the upper endplate was $4.2 \mathrm{~mm}$ (range, 2-5 $\mathrm{mm})$. However, Shimizu et al. [24] found that the bifurcation level of the segmental arteries was different at each level. Orita et al. [17] evaluated the segmental artery using magnetic resonance sagittal plane images and reported that the segmental arteries branched upward at L1-3 but branched downward at L4-5. However, the detailed vascular anatomy of the anterior part of the spinal column has not been previously reported.

In this study, we examined the anatomy of the segmental arteries and veins in the anterior part of the vertebral column using five human cadaveric specimens. The proportion of the segmental arteries and veins that intersected the anterior part of the IVD was high (artery, 28.1\%; vein, $42.1 \%)$. Fig. 3 shows that the horizontal distance between the lateral edge of the caudal adjacent IVD and intersection point of the segmental vessels is approximately 
$\geq 10 \mathrm{~mm}$, but in a few cases, we found segmental vessels close to the edge of the IVD. For example, the right L3 segmental vein intersected the IVD only $1.3 \mathrm{~mm}$ from the lateral edge. Therefore, one should pay careful attention to the depth of the anterior retractor when performing LLIF.

The proportion of the arteries and veins that intersect the IVD in the anterior part increases at higher lumbar levels. Particularly, the proportions of L2 segmental arteries, L2 veins, and L3 veins that intersected the IVD in our study were $70 \%, 60 \%$, and $50 \%$, respectively. We consider that this is because the segmental arteries and veins originate more distally at higher IVD levels. The higher IVD levels have steeper lumbar segmental arteries that branch toward the cranial side. That is, the higher lumbar segmental vessels originate caudally and run cranially over the IVD. Shimizu et al. [24] reported that, in five adult cadaveric specimens, the segmental arteries originated just below the corresponding vertebra except for the last two lumbar arteries that originated from the center of the corresponding vertebrae. They considered that the dissociation of the origin of the segmental arteries and the corresponding vertebrae could be due to differences in the longitudinal growth between the vertebral column and aorta. Originally, in the human embryo, segmental arteries belonging to each somite arise from the dorsal aorta at approximately 3 weeks of gestation, and their origins are located at the same levels as their corresponding somite [26]. Therefore, this difference in growth speed could be responsible for the variations in the origins of the segmental arteries. However, in the lower lumbar levels, the origin of the segmental arteries might remain at the same level as the corresponding IVD because the lower abdominal aorta is less mobile. In addition to growth speed, variation in the tortuosity of the abdominal aorta with age might explain the differences between the origins of the segmental arteries and veins and their corresponding IVD [27].

In comparing the right and left segmental vessels, we found that the right segmental arteries intersected the IVD in six of 16 cases (37.5\%), and the left arteries intersected the IVD in three of 16 cases $(18.8 \%)$. The right segmental veins intersected the IVD in five of nine cases (55.6\%), and the left veins intersected the IVD in three of 10 cases $(30.0 \%)$. Although these were not statistically significant, the right segmental vessels intersected the IVD more frequently than the left segmental vessels. This suggests that surgical approaches from the right side require more careful attention. A limitation of this study is the small sample size, which made it more difficult to confirm statistically significant differences.

Our results indicated that segmental vessels can intersect the IVD, especially at higher lumbar levels. We suggest that, at higher lumbar levels, each patient's individual anatomy should be studied preoperatively, particularly in relation to the segmental vessels. Spinal CT angiography is an effective method to evaluate the segmental arteries [19], but the segmental veins are difficult to visualize. Therefore, it is important to assume that some segmental veins intersect the IVD at the anterior part of vertebra during surgery.

Taking these results into account, segmental vessels can intersect the IVD, especially at higher lumbar levels. When placing an anterior retractor during LLIF or incising the anterior longitudinal ligament during ACR, one should fully understand the vascular anatomy of the anterior aspect of the spinal column. Therefore, it is useful to sufficiently examine the course of the segmental vessels to achieve good outcomes following LLIF and ACR. A 3D reconstruction image using spinal CT angiography can be used preoperatively to examine the anatomy of the segmental arteries of each patient [19]. However, it is difficult to completely visualize the segmental veins. Therefore, it is important to understand that some segmental veins intersect the IVD. Vascular complications must be prevented to achieve successful LLIF and ACR.

\section{Conclusions}

The proportion of segmental arteries and veins that intersect the IVD in the anterior part of the spinal column is high. At higher IVD levels, more segmental arteries and veins intersect the caudal part of the adjacent IVD. Therefore, it is necessary to consider these individual anatomical features to prevent vascular damage during LLIF surgery.

\section{Conflict of Interest}

No potential conflict of interest relevant to this article was reported.

\section{Acknowledgments}

The authors sincerely appreciate Prof. Ueki from the 
Department of Integrative Anatomy, Graduate School of Medical Sciences, Nagoya City University, Nagoya.

\section{References}

1. Mayer HM. A new microsurgical technique for minimally invasive anterior lumbar interbody fusion. Spine (Phila Pa 1976) 1997;22:691-9.

2. Kaneda K, Shono Y, Satoh S, Abumi K. New anterior instrumentation for the management of thoracolumbar and lumbar scoliosis: application of the Kaneda two-rod system. Spine (Phila Pa 1976) 1996;21:125061.

3. Kim WJ, Lee JW, Park KY, Chang SH, Song DG, Choy WS. Treatment of adult spinal deformity with sagittal imbalance using oblique lumbar interbody fusion: can we predict how much lordosis correction is possible? Asian Spine J 2019;13:1017-27.

4. Nakashima H, Kanemura T, Satake K, et al. Comparative radiographic outcomes of lateral and posterior lumbar interbody fusion in the treatment of degenerative lumbar kyphosis. Asian Spine J 2019;13:395402.

5. Acosta FL, Liu J, Slimack N, Moller D, Fessler R, Kos$\mathrm{ki}$ T. Changes in coronal and sagittal plane alignment following minimally invasive direct lateral interbody fusion for the treatment of degenerative lumbar disease in adults: a radiographic study. J Neurosurg Spine 2011;15:92-6.

6. Rodgers WB, Gerber EJ, Patterson J. Intraoperative and early postoperative complications in extreme lateral interbody fusion: an analysis of 600 cases. Spine (Phila Pa 1976) 2011;36:26-32.

7. Nakashima H, Kanemura T, Satake K, et al. Indirect decompression using lateral lumbar interbody fusion for restenosis after an initial decompression surgery. Asian Spine J 2020;14:305-11.

8. Beng TB, Kotani Y, Sia U, Gonchar I. Effect of indirect neural decompression with oblique lateral interbody fusion was influenced by preoperative lumbar lordosis in adult spinal deformity surgery. Asian Spine J 2019;13:809-14.

9. Wang MY, Vasudevan R, Mindea SA. Minimally invasive lateral interbody fusion for the treatment of rostral adjacent-segment lumbar degenerative stenosis without supplemental pedicle screw fixation. J Neurosurg Spine 2014;21:861-6.
10. Akbarnia BA, Mundis GM Jr, Moazzaz P, et al. Anterior column realignment (ACR) for focal kyphotic spinal deformity using a lateral transpsoas approach and ALL release. J Spinal Disord Tech 2014;27:29-39.

11. Deukmedjian AR, Le TV, Baaj AA, Dakwar E, Smith DA, Uribe JS. Anterior longitudinal ligament release using the minimally invasive lateral retroperitoneal transpsoas approach: a cadaveric feasibility study and report of 4 clinical cases. J Neurosurg Spine 2012;17:530-9.

12. Saigal R, Mundis GM Jr, Eastlack R, Uribe JS, Phillips FM, Akbarnia BA. Anterior column realignment (ACR) in adult sagittal deformity correction: technique and review of the literature. Spine (Phila $\mathrm{Pa}$ 1976) 2016;41 Suppl 8:S66-73.

13. Fujibayashi S, Kawakami N, Asazuma T, et al. Complications associated with lateral interbody fusion: nationwide survey of 2998 cases during the first 2 years of its use in Japan. Spine (Phila Pa 1976) 2017;42:1478-84.

14. Kueper J, Fantini GA, Walker BR, Aichmair A, Hughes AP. Incidence of vascular complications during lateral lumbar interbody fusion: an examination of the mini-open access technique. Eur Spine J 2015;24:800-9.

15. Takata Y, Sakai T, Tezuka F, et al. Risk assessment of lumbar segmental artery injury during lateral transpsoas approach in the patients with lumbar scoliosis. Spine (Phila Pa 1976) 2016;41:880-4.

16. Davis TT, Hynes RA, Fung DA, et al. Retroperitoneal oblique corridor to the L2-S1 intervertebral discs in the lateral position: an anatomic study. J Neurosurg Spine 2014;21:785-93.

17. Orita S, Inage $\mathrm{K}$, Sainoh $\mathrm{T}$, et al. Lower lumbar segmental arteries can intersect over the intervertebral disc in the oblique lateral interbody fusion approach with a risk for arterial injury: radiological analysis of lumbar segmental arteries by using magnetic resonance imaging. Spine (Phila Pa 1976) 2017;42:13542.

18. Nojiri H, Miyagawa K, Banno S, et al. Lumbar artery branches coursing vertically over the intervertebral discs of the lower lumbar spine: an anatomic study. Eur Spine J 2016;25:4195-8.

19. Heo DH, Cho YJ, Sheen SH, Hong MS, Cho SM, Park $\mathrm{SH}$. 3D reconstructions of spinal segmental arteries using CT angiography: applications in minimally 
invasive spinal procedures. AJNR Am J Neuroradiol 2010;31:1635-9.

20. Tezuka F, Sakai T, Nishisho T, et al. Variations in arterial supply to the lower lumbar spine. Eur Spine J 2016;25:4181-7.

21. Abe K, Orita S, Mannoji C, et al. Perioperative complications in 155 patients who underwent oblique lateral interbody fusion surgery: perspectives and indications from a retrospective, multicenter survey. Spine (Phila Pa 1976) 2017;42:55-62.

22. Beckman JM, Vincent B, Park MS, et al. Contralateral psoas hematoma after minimally invasive, lateral retroperitoneal transpsoas lumbar interbody fusion: a multicenter review of 3950 lumbar levels. J Neurosurg Spine 2017;26:50-4.

23. Peiro-Garcia A, Dominguez-Esteban I, Alia-Benitez J. Retroperitoneal hematoma after using the extreme lateral interbody fusion (XLIF) approach: presentation of a case and a review of the literature. Rev Esp
Cir Ortop Traumatol 2016;60:330-4.

24. Shimizu S, Tanaka R, Kan S, Suzuki S, Kurata A, Fujii K. Origins of the segmental arteries in the aorta: an anatomic study for selective catheterization with spinal arteriography. AJNR Am J Neuroradiol 2005;26:922-8.

25. Alkadhim M, Zoccali C, Abbasifard S, et al. The surgical vascular anatomy of the minimally invasive lateral lumbar interbody approach: a cadaveric and radiographic analysis. Eur Spine J 2015;24 Suppl 7:906-11.

26. Patten BM. The development of the heart. In: Gould SE, editor. Pathology of the heart and blood vessels. 3rd ed. Springfield (IL): Charles C. Thomas Publisher; 1968. p. 20-90.

27. Dougherty G, Varro J. A quantitative index for the measurement of the tortuosity of blood vessels. Med Eng Phys 2000;22:567-74. 